\title{
Neuroendoscopic Evacuation for Spontaneous Cerebellar Hemorrhage Is a Safe and Secure Approach and May Become a Mainstream Technique
}

\author{
Hideki Atsumi, ${ }^{1}$ Tanefumi BabA, ${ }^{1}$ Azusa SunAGA, ${ }^{1}$ Yumetaro SAKaKIBARA, ${ }^{1}$ \\ Yoichi NONAKA, ${ }^{1}$ Takatoshi SORIMACHI, ${ }^{1}$ and Mitsunori MATSUMAE ${ }^{1}$ \\ ${ }^{1}$ Department of Neurosurgery, Tokai University School of Medicine, Isehara, \\ Kanagawa, Japan
}

\begin{abstract}
Patients with spontaneous cerebellar hemorrhage present with rapidly deteriorating neurological symptoms due to a hematoma-induced mass effect in the brainstem. We compared the standard surgical approach of a suboccipital craniectomy with neuroendoscopic surgery for treating spontaneous cerebellar hemorrhage. We performed a retrospective analysis of 41 patients indicated for surgery to treat spontaneous cerebellar hemorrhage. At our hospital, craniectomy was performed until 2010, and neuroendoscopic surgery was performed thereafter when a qualified surgeon was available. Duration of surgery and intraoperative blood loss were lower in the neuroendoscopic surgery group. The extent of hematoma removal and the percentage of patients requiring shunting were similar between groups. The mass effect was resolved in all patients in both groups, and no substantial re-bleeding was observed in either group. The outcomes at discharge were comparable between the two groups. Our surgeons used the supine lateral position, which involves fewer burdens to the patient than the prone position. Selection of the site of the burr hole is important to avoid the midline and to avoid the area exactly above the transverse and sigmoid sinus. Our results suggest that minimally invasive neuroendoscopic surgery is safe and superior to craniectomy due to shortened duration of surgery and decreased intraoperative bleeding.
\end{abstract}

Key words: cerebellar hemorrhage, neuroendoscopic surgery, minimally invasive surgery

\section{Introduction}

Spontaneous cerebellar hemorrhage involves a smaller volume of the posterior fossa compared with spontaneous supratentorial hemorrhage. Consequently, some patients with this condition present with rapidly deteriorating neurological symptoms due to a hematoma-induced mass effect in the brainstem as well as obstructive hydrocephalus. ${ }^{1-5)}$ The American Heart Association/ American Stroke Association guidelines ${ }^{6}$ recommend a $^{2}$ surgical hematoma evacuation procedure with Class I level evidence in hematomas $\geq 3 \mathrm{~cm}$ in diameter or in patients presenting with brainstem compression and/or hydrocephalus. Thus, a hematoma evacuation procedure is proactively performed in indicated patients. Some common causes of cerebellar hemorrhage include hypertension, aneurysms that develop in the

Received May 29, 2019; Accepted August 5, 2019

Copyright@ 2019 by The Japan Neurosurgical Society This work is licensed under a Creative Commons AttributionNonCommercial-NoDerivatives International License. distal part of the superior cerebellar artery or posterior inferior cerebellar artery, a small nidus of arteriovenous malformation, dural arteriovenous fistula, and cavernous malformation. For these reasons, computed tomography (CT)-guided stereotactic aspiration or neuroendoscopic surgery are delayed in spontaneous cerebellar hemorrhage compared with supratentorial intracerebral hemorrhage. Until now, with consideration for safety (i.e., definitive management of the bleeding point) and anatomical characteristics in which the posterior fossa has a small volume, surgeons have traditionally performed hematoma evacuation through a suboccipital craniotomy and external decompression as standard treatment. However, advancements in diagnostic imaging techniques such as CT and magnetic resonance imaging have resulted in a more precise preoperative diagnosis and have markedly improved the safety of minimally invasive treatments using neuroendoscopy. We therefore postulated that comparing suboccipital craniectomy and neuroendoscopic surgery approaches for treating spontaneous cerebellar hemorrhage is necessary. Thus, we conducted 
a retrospective study to investigate the preoperative, interoperative, and postoperative factors.

\section{Patients and Methods}

We investigated 41 patients who were admitted to the authors' institution between January 2008 and November 2018 with a surgical indication for cerebellar hemorrhage. Patients with a secondary cerebellar hemorrhage that was related to an aneurysm, arteriovenous malformation, venous anomaly, or tumor were excluded from this study. Four patients taking direct oral anticoagulants and warfarin were excluded because antagonists were marketed in our country during the observation period, and the intake of these medications may have significantly modulated the results. In this retrospective study, we investigated factors such as sex, age, initial Glasgow Coma Scale, ${ }^{7)}$ hematoma volume calculated with $\mathrm{CT}{ }^{8)}$ history of taking antiplatelet agent medication, initial CT findings, duration of surgery, intraoperative blood loss, external drainage, shunting, postoperative CT findings, complications, duration of hospital stay (days), and patient outcome at discharge based on the modified Rankin Scale (mRS). $\left.{ }^{9}\right)$ The Mann-Whitney $U$-test was used for statistical analysis of the duration of hospital stay, duration of surgery, and intraoperative blood loss (IBM SPSS version 23, IBM, Tokyo, Japan). Table 1 shows the patient characteristics. Surgery for spontaneous cerebellar hemorrhage was indicated for a diameter of $3 \mathrm{~cm}$ or greater on CT with exacerbated neurological signs, or hydrocephalus caused by fourth ventricle obstruction due to spontaneous cerebellar hemorrhage. Craniectomy was performed until 2010, and neuroendoscopic surgery was performed thereafter when a qualified neuroendoscopic surgeon certified by the Japanese Society for Neuroendoscopy was available to perform an emergency operation.

Hematoma evacuation with suboccipital craniectomy was performed using a standard procedure. The extent of craniectomy and the decision to remove the posterior arch of $\mathrm{C} 1$ were determined by the surgeon.

Two qualified neuroendoscopic surgeons were in charge of the hematoma evacuation with neuroendoscopy. All endoscopic surgeries were performed using a navigation system. After induction of general anesthesia with tracheal intubation, the patient was placed in a supine lateral position as shown in Fig. 1. Specifically, the shoulder on the affected

Table 1 Characteristics of patients in this study

\begin{tabular}{lcc}
\hline & Endoscopy & Craniectomy \\
\hline Number of patients & 15 & 22 \\
Female/male & $10 / 5$ & $7 / 15$ \\
Mean age (years, range) & $73(61-86)$ & $66(51-81)$ \\
Mean GCS (range) & $10(4-14)$ & $9.2(3-15)$ \\
$\begin{array}{l}\text { Mean hematoma volume } \\
\text { (ml, range) }\end{array}$ & $21.8(14-45)$ & $25.1(9-55)$ \\
$\begin{array}{l}\text { Taking an antiplatelet } \\
\text { agent (Number of patients) }\end{array}$ & 2 & 2 \\
$\begin{array}{l}\text { Hematoma occupying the } \\
\text { IVth ventricle on initial } \\
\text { CT (Number of patients) }\end{array}$ & 11 & 17 \\
$\begin{array}{l}\text { Hematoma occupying the } \\
\text { IIIrd ventricle on initial } \\
\text { CT (Number of patients) }\end{array}$ & 6 & 6 \\
$\begin{array}{l}\text { Acute obstructive } \\
\text { hydrocephalus (\%) }\end{array}$ & 100 & \\
\hline
\end{tabular}

CT: computed tomography, GCS: Glasgow Coma Scale.
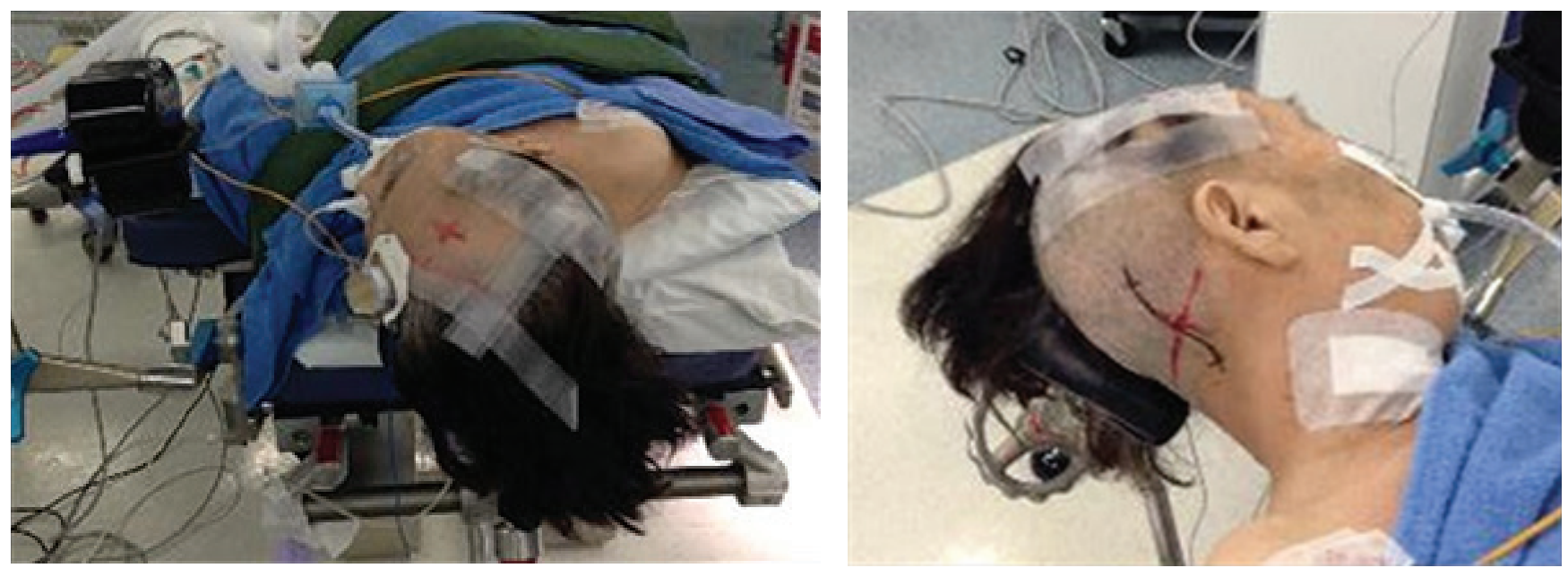

Fig. 1 Positioning of the patient for endoscopic surgery for spontaneous cerebellar hemorrhage. This figure shows the body position for the surgery to treat a right cerebellar hemorrhage. The shoulder of the affected side is slightly raised, and the head is rotated toward the unaffected side. 
side was elevated by inserting a pillow underneath, and the head was rotated away from the hematoma site to prevent congestion of venous drainage; as a result, the insertion direction of the endoscope was parallel to the operating table, facilitating an easier approach to the hematoma in the supine position. First, a ventricular catheter was inserted into the lateral ventricle and was clamped until hematoma removal. A burr hole was created at a safe site after confirming the positions of the transverse and sigmoid sinuses as well as the location of the hematoma using the navigation system. As a result, the burr hole was often created slightly lateral to the midpoint of the inion and tip of the mastoid process, immediately below the superior nuchal line. After incising the dura mater, the location of the hematoma was re-confirmed with a navigation system, and a transparent sheath (NeuroPort: Olympus Medical Systems Corp., Tokyo, Japan, outer diameter $10 \mathrm{~mm}$ ) was inserted. Subsequently, a rigid endoscope was inserted through the sheath (2.7 mm diameter, MS1000L Machida Endoscope Co., Ltd., Abiko, Chiba, Japan) to perform hematoma evacuation while simultaneously confirming the hematoma. Definitive coagulation under endoscopic visualization was performed during hematoma evacuation. In the operative strategy, we avoided forceful removal of a hematoma that had extended into the fourth ventricle. After determining sufficient hematoma removal, observed cerebrospinal fluid was drained from the fourth ventricle into the cavity resulting from hematoma removal; full hemostasis was confirmed; the dura mater, muscle layer, and skin were sutured; and the surgery was concluded.

This study was approved by the ethics committee of our institution (titled "Clinical study on enlargement of intracerebral hemorrhage" accepted on March 2, 2017; No. 16R-264.)

\section{Results}

Significantly different factors between the neuroendoscopy group and the craniectomy group were duration of surgery and intraoperative blood loss, revealing the minimally invasive characteristics of neuroendoscopy (Table 2). The outcomes at discharge were comparable between the two groups (Table 3). Our result do not show an improvement in the functional prognosis in the endoscopic group. We conclude that the survival rate of the neuroendoscopy group was similar to that of the craniectomy group (Table 3). The percentage of patients with acute obstructive hydrocephalus and the extent of intraventricular hematoma removal were examined by comparing the percentage of hematoma occupation
Table 2 Surgical results in this study

\begin{tabular}{|c|c|c|}
\hline & Endoscopy & Craniectomy \\
\hline $\begin{array}{l}\text { Mean duration of } \\
\text { surgery }(\mathrm{min}) \pm \mathrm{SD}\end{array}$ & $121.4 \pm 42.5^{*}$ & $241.9 \pm 64.4$ \\
\hline $\begin{array}{l}\text { Mean intraoperative } \\
\text { bleeding }(\mathrm{mL}) \pm \mathrm{SD}\end{array}$ & $59.66 \pm 48.06^{* *}$ & $294.2 \pm 271.02$ \\
\hline $\begin{array}{l}\text { External ventricular } \\
\text { drainage required } \\
\text { (Number of patients) }\end{array}$ & 13 & 22 \\
\hline $\begin{array}{l}\text { Shunting procedure } \\
\text { required (Number of } \\
\text { patients) }\end{array}$ & 3 & 4 \\
\hline $\begin{array}{l}\text { Hematoma } \\
\text { occupying the } \\
\text { IVth ventricle on } \\
\text { postoperative CT } \\
\text { (Number of patients) }\end{array}$ & $0 / 11^{* * *}$ & $0 / 17^{* * *}$ \\
\hline $\begin{array}{l}\text { Hematoma } \\
\text { occupying the } \\
\text { IIIrd ventricle on } \\
\text { postoperative CT } \\
\text { (Number of patients) }\end{array}$ & $3 / 6^{* * *}$ & $2 / 6^{* * *}$ \\
\hline $\begin{array}{l}\text { Additional surgery } \\
\text { required (Number of } \\
\text { patients) }\end{array}$ & 0 & 3 \\
\hline $\begin{array}{l}\text { Infection (Number of } \\
\text { patients) }\end{array}$ & 1 & 2 \\
\hline $\begin{array}{l}\text { CSF leakage (Number } \\
\text { of patients) }\end{array}$ & 0 & 4 \\
\hline $\begin{array}{l}\text { Duration of hospital } \\
\text { stay (days) }\end{array}$ & $62 \pm 8$ & $83 \pm 18$ \\
\hline $\begin{array}{l}\text { Removal rate of } \\
\text { hematoma (\%) }\end{array}$ & $95 \%$ & $90 \%$ \\
\hline
\end{tabular}

Table 3 Clinical outcome in this study

\begin{tabular}{lccc}
\hline Modified & Endoscopy & & Craniectomy \\
\cline { 2 - 2 } & Numbin Scale of patients & & Number of patients \\
\hline 0 & 0 & 0 \\
1 & 0 & 0 \\
2 & 0 & 1 \\
3 & 1 & 6 \\
4 & 8 & 6 \\
5 & 6 & 9 \\
6 & 1 & 0 \\
\hline
\end{tabular}

of the ventricle; no significant differences were observed between the two groups (Tables 1 and 2). Moreover, we found no differences between the two groups in the percentage of patients requiring 
shunting, the removal rate of the hematoma, or the duration of the hospital stay (Table 2). Subcutaneous cerebrospinal fluid retention or cerebrospinal fluid leakage from the wound was observed in four patients in the craniectomy group, and three of these patients required an additional surgery to correct the cerebrospinal fluid leakage (Table 2). Of these, one patient in the neuroendoscopy group developed meningitis. Two patients in the craniectomy group developed wound infections. In all patients in both groups, postoperative CT confirmed that removal of the hematoma was satisfactory (Table 2), and the mass effect had resolved. Re-bleeding that was substantial enough to consider another hematoma evacuation procedure was not observed in either group. As a result, the median mRS was 4 in the endoscopic group and 4 in the craniectomy group; no significant differences were seen in outcome at discharge according to the mRS between groups (Table 3). One patient in the neuroendoscopy group who had a mRS of 6 died from malignant pleural effusion, which was caused by an existing malignant disease and was unrelated to spontaneous cerebellar hemorrhage.

Figure 2 shows images of a typical patient. This 84-year-old man, who had been taking antiplatelet agents to treat cerebral infarction, was also diagnosed with Alzheimer's dementia. His Glasgow
Coma Scale was 1-1-2 at admission to our hospital, and the patient presented with sluggish light reflex along with a decerebrate position. CT confirmed a spontaneous cerebellar hemorrhage that extended into fourth ventricle and obstructive hydrocephalus (Fig. 2, upper panel). A hematoma evacuation with neuroendoscopy was performed. Postoperative CT revealed satisfactory hematoma evacuation (Fig. 2, lower panel), and the patient was transferred to another hospital with a mRS of 5 .

Figure 3 shows the selection of the surgical procedure to treat spontaneous cerebellar hemorrhage at our hospital from 2008 to 2018. The selection of neuroendoscopy has been rising over time, and this is largely attributed to the increase in the number of qualified neuroendoscopic surgeons.

\section{Discussion}

This study found that neuroendoscopic surgery is minimally invasive and is superior to craniectomy due to shortened duration of surgery and decreased intraoperative bleeding.

Our study has several limitations. First, this was not a randomized controlled study. Second, the craniectomy group involved various surgeons including neurosurgeons-in-training. In contrast, the neuroendoscopy group included only two qualified
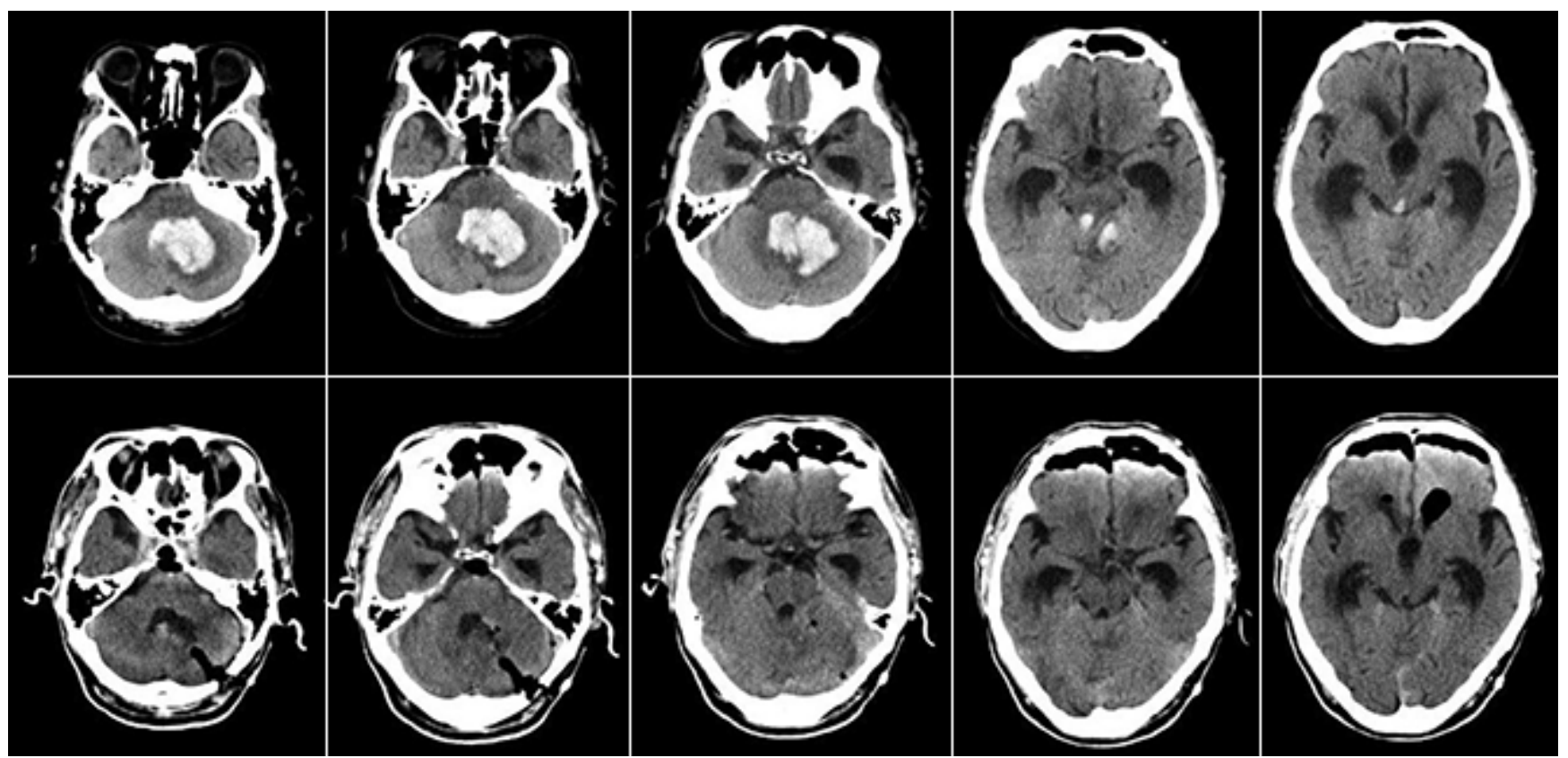
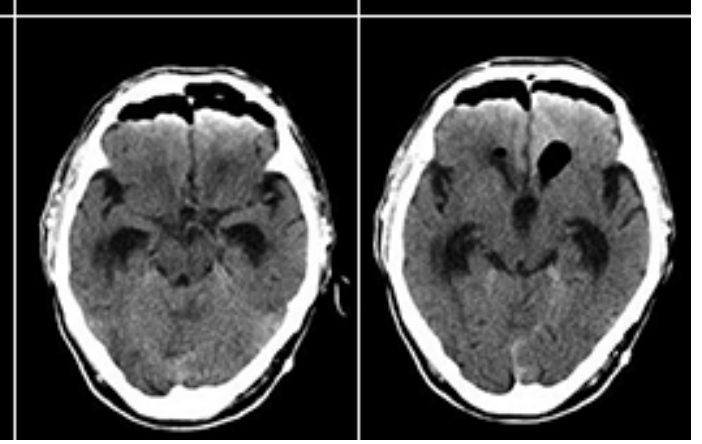

Fig. 2 Illustration of a case of neuroendoscopic surgery for spontaneous cerebellar hemorrhage. An 84-year-old man, while taking an antiplatelet agent, he developed consciousness disturbance. Upper panel: The hematoma in the right cerebellar hemisphere has extended into the fourth ventricle, and non-communicating hydrocephalus is present. Lower panel: A burr hole was created in the right suboccipital region. The hematoma was removed in a satisfactory manner using neuroendoscopy, and the ventricular enlargement was alleviated. 


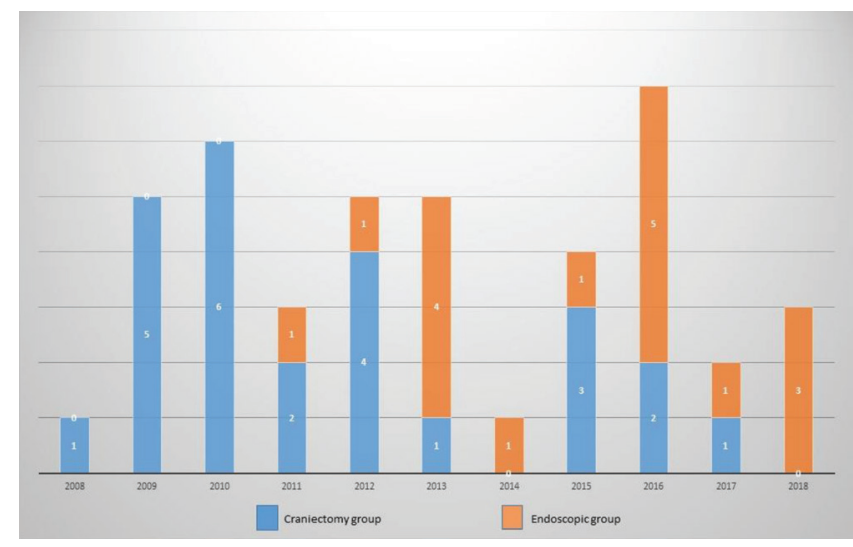

Fig. 3 Trend in selection of the surgical procedure for spontaneous cerebellar hemorrhage at our hospital. Percentages of selection of neuroendoscopy and craniectomy from 2008 to 2018 are shown. Neuroendoscopy was selected more frequently in recent years.

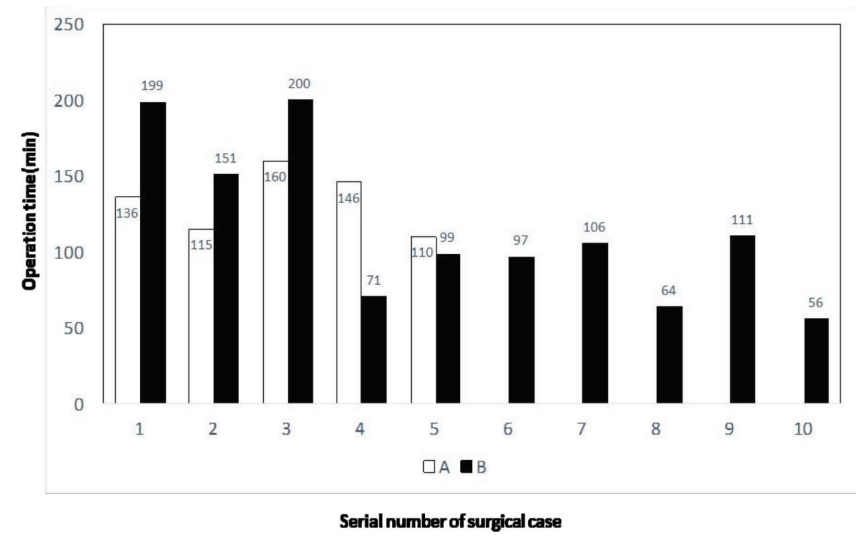

Fig. 4 Trend in duration of surgery for spontaneous cerebellar hemorrhage by two surgeons. Although the duration of surgery was long for many surgeries performed immediately after the introduction of neuroendoscopy, it has shortened over time, especially for surgeon B who has more experience.

neuroendoscopic surgeons who performed the surgery, thereby introducing a bias. This may have contributed to the increase in standard deviation in intraoperative blood loss and duration of surgery as well as the increase in complications and additional surgeries in the craniectomy group. Third, this was a single center study. Fourth, this study included the early stage of spontaneous cerebellar hemorrhage surgeries performed by qualified neuroendoscopic surgeons who started endoscopic surgery. The duration of surgery was longer in these operations in the beginning. Figure 4 shows the changes in duration of surgery by the two qualified neuroendoscopic surgeons, displaying their process of experience and duration of surgery. Surgeon B, who has performed many neuroendoscopic surgeries, showed a shortened duration of surgery with increasing experience in neuroendoscopy. Some of the more recent surgeries have required $<1 \mathrm{~h}$. Based on the duration of the hematoma evacuation procedure for spontaneous cerebellar hemorrhage, neuroendoscopy is sufficiently superior compared with craniectomy. Additionally, bleeding mostly occurred from the muscle layer, bone edge, or emissary vein sinus during the craniotomy. These types of unnecessary blood loss led to increased intraoperative bleeding in the craniectomy group. On the other hand, burr hole surgery can minimize such unnecessary blood loss.

Surgical treatment for spontaneous cerebellar hemorrhage is recommended in the guidelines ${ }^{6)}$ for hematomas larger than $3 \mathrm{~cm}$ in diameter, or in cases with exacerbated neurological symptoms due to hematoma-induced brainstem compression and hydrocephalus. This is based on well-known reports by Da Pian et al., ${ }^{10)}$ Firsching et al., ${ }^{11)}$ and van Loon et al. ${ }^{12)}$ that showed that surgical treatments, such as hematoma evacuation and surgical decompression, result in better patient outcomes than medical treatment in patients demonstrating neurological deterioration. However, these criteria are unfortunately based on non-randomized studies. We therefore sought to compare craniectomy and neuroendoscopic surgery for treating spontaneous cerebellar hemorrhage. To the best of our knowledge, only one study has compared neuroendoscopy and craniectomy in spontaneous cerebellar hemorrhage. Yamamoto et al. ${ }^{13)}$ concluded that shunting was performed less frequently in the neuroendoscopy group and that the duration of surgery and amount of blood loss were superior in the neuroendoscopy group compared to the craniectomy group. Nishihara et al. compared a neuroendoscopy group $(n=3)$ with a CT-guided stereotactic surgery group $(n=3)$ and found that although the duration of surgery was similar between the two groups, the neuroendoscopy group had better hematoma evacuation rates and better outcomes. However, this report compared two groups with different ages and neurological grades at admission.

With consideration for the aforementioned biases, our treatments showed impressive life-saving results that is considered to be an inevitable trend in surgical treatment compared with non-surgical cases when considering cases with more severe neurological deterioration. Our results demonstrated the superiority of neuroendoscopy for spontaneous cerebellar hemorrhage evacuation from the perspective of both duration of surgery and intraoperative blood loss. These results corroborate the findings by Nishihara et al., ${ }^{14,15)}$ who also demonstrated the minimally 
invasive nature of neuroendoscopy. Furthermore, none of the patients in the neuroendoscopy group exhibited re-bleeding. Although this is equivalent to bipolar hemostasis performed under a microscope during surgery, it shows the advantage of being able to perform definitive hemostasis while directly visualizing the bleeding point with endoscopy when using various types of coagulation instruments, as reported by Nishihara et al. ${ }^{14)}$ In this investigation, none of the patients required surgical decompression due to increased brain edema around the hematoma or ischemia after neuroendoscopic surgery. Similarly, Yamamoto et al. ${ }^{13)}$ and Nishihara et al. ${ }^{15)}$ also did not report patients who required additional surgery due to the above reasons. Furthermore, patients rarely require posterior cranial fossa decompression after CT-guided stereotactic surgery. ${ }^{16)}$ In other words, the results raised questions about whether external decompression is required for surgical treatment of spontaneous cerebellar hemorrhage. Figure 3 shows the transition in surgical methods at our institution over the past 10 years. A recent trend is to select neuroendoscopic surgery. This reflects a consensus that was formed among affiliated hospital surgeons that neuroendoscopic surgeries are minimally invasive.

In this study, we did not observe a difference in the extent of resolution of intraventricular hematoma between the craniectomy group and neuroendoscopy group, indicating that intraventricular hematoma evacuation can be achieved with neuroendoscopic surgery similarly to craniectomy. Nonaka et al. ${ }^{17)}$ described a case in which spontaneous cerebellar hemorrhage evacuation with neuroendoscopy was combined with supratentorial intraventricular hematoma evacuation at same time. Although these authors did not discuss the duration of surgery, addition of an endoscopic approach as necessary to the cerebellar hematoma evacuation procedure may be a more aggressive treatment method for patients presenting with a marked intraventricular hematoma in the supratentorial region. This simultaneous approach to remove both infra and supratentorial hematomas is controversial in the field of neurosurgery.

Next, we will discuss anesthesia, positioning, and the site of the burr hole. In our hospital, inhalation anesthesia was performed in four cases of craniotomy in 2008 and 2009. Thereafter, all cases including endoscopic surgery were performed under total intravenous anesthesia. No difference was found according to the method of anesthesia. Although Yamamoto et al. performed the surgery in a lateral position, neuroendoscopic surgery can also be performed in the supine lateral position (Fig. 1). The lateral or supine lateral position, which can be chosen by the surgeon, involves fewer burdens to the patient than the prone position. There are several reasons for this as follows: first, an extensive craniotomy is not necessary; second, the labor to change the body position is eliminated, and the duration of anesthesia is shortened; and third, older patients benefit from this when considering their respiratory and circulatory dynamics. Thus, these positions are significantly more advantageous compared with craniectomy in the prone position. However, a supine lateral position can lead to disorientation by beginner surgeons, indicating the need to be cautious with the selection of the burr hole site, direction of ventricular catheter insertion, and approach for hematoma removal. For the selection of the burr hole in neuroendoscopic surgery, we avoid the midline where the occipital bone is thick and protrudes toward the dura mater in a convex manner and where a remnant in the occipital sinus is problematic. In addition, to avoid a burr hole exactly above the transverse and sigmoid sinus, the use of a navigation system is desirable from a safety perspective. Moreover, the structure of the posterior cranial fossa (such as the angle of the cerebellar tentorium) varies widely, and a navigation system is therefore useful for confirming the direction of hematoma extension and the depth to complete the hematoma extraction. This system is also beneficial for avoiding serious complications such as brainstem injury and should be used whenever possible.

\section{Conclusion}

We found no differences in outcomes between the craniectomy group and neuroendoscopy group. Spontaneous cerebellar hemorrhage treatment using neuroendoscopy, which is performed by a highly trained qualified neuroendoscopic surgeon, resulted in a shorter duration of surgery and a smaller volume of intraoperative bleeding, and was therefore determined to be superior as a minimally invasive and safe technique. Our findings suggested that neuroendoscopy may become a mainstream technique in lieu of the traditional craniectomy in surgeries to treat spontaneous cerebellar hemorrhage. However, very few reports have been published about neuroendoscopic surgery for spontaneous cerebellar hemorrhage, indicating the need to promote a multicenter study with a larger sample size to validate the safety and effectiveness of this procedure.

\section{Conflicts of Interest Disclosure}

The authors declare no conflicts of interest. 


\section{References}

1) Ironside N, Chen CJ, Dreyer V, Christophe B, Buell TJ, Connolly ES: Location-specific differences in hematoma volume predict outcomes in patients with spontaneous intracerebral hemorrhage. Int J Stroke 1747493019830589, 2019

2) Troberg E, Kronvall E, Hansen BM, Nilsson OG: Prediction of long-term outcome after intracerebral hemorrhage surgery. World Neurosurg pii: S18788750(18)32834-1, 2018

3) Steiner T, Al-Shahi Salman R, Beer R, et al.: European Stroke Organisation (ESO) guidelines for the management of spontaneous intracerebral hemorrhage. Int J Stroke 9: 840-855, 2014

4) Mathew P, Teasdale G, Bannan A, Oluoch-Olunya D: Neurosurgical management of cerebellar haematoma and infarct. $J$ Neurol Neurosurg Psychiatry 59: 287-292, 1995

5) Chen R, Wang X, Anderson CS, et al.: Infratentorial intracerebral hemorrhage. Stroke 50: 1257-1259, 2019

6) Hemphill JC, Greenberg SM, Anderson CS, et al.: Guidelines for the Management of Spontaneous Intracerebral Hemorrhage: A Guideline for Healthcare Professionals From the American Heart Association/ American Stroke Association. Stroke 46: 2032-2060, 2015

7) Teasdale G, Jennett B: Assessment of coma and impaired consciousness. A practical scale. Lancet 2: 81-84, 1974

8) Niizuma H, Suzuki J: Stereotactic aspiration of putaminal hemorrhage using a double track aspiration technique. Neurosurgery 22: 432-436, 1988

9) van Swieten JC, Koudstaal PJ, Visser MC, Schouten $\mathrm{HJ}$, van Gijn J: Interobserver agreement for the assessment of handicap in stroke patients. Stroke 19: 604-607, 1988
10) Da Pian R, Bazzan A, Pasqualin A: Surgical versus medical treatment of spontaneous posterior fossa haematomas: a cooperative study on 205 cases. Neurol Res 6: 145-151, 1984

11) Firsching R, Huber M, Frowein RA: Cerebellar haemorrhage: management and prognosis. Neurosurg Rev 14: 191-194, 1991

12) van Loon J, Van Calenbergh F, Goffin J, Plets C: Controversies in the management of spontaneous cerebellar haemorrhage. A consecutive series of 49 cases and review of the literature. Acta Neurochir (Wien) 122: 187-193, 1993

13) Yamamoto T, Nakao Y, Mori K, Maeda M: Endoscopic hematoma evacuation for hypertensive cerebellar hemorrhage. Minim Invasive Neurosurg 49: 173-178, 2006

14) Nishihara T, Nagata K, Tanaka $S$, et al.: Newly developed endoscopic instruments for the removal of intracerebral hematoma. Neurocrit Care 2: 67-74, 2005

15) Nishihara T, Morita A, Teraoka A, Kirino T: Endoscopyguided removal of spontaneous intracerebral hemorrhage: comparison with computer tomographyguided stereotactic evacuation. Childs Nerv Syst 23: 677-683, 2007

16) Mohadjer M, Eggert R, May J, Mayfrank L: CT-guided stereotactic fibrinolysis of spontaneous and hypertensive cerebellar hemorrhage: long-term results. J Neurosurg 73: 217-222, 1990

17) Nonaka M, Yagi K, Abe H, et al.: Endoscopic surgery via a combined frontal and suboccipital approach for cerebellar hemorrhage. Surg Neurol Int 9: 68, 2018

Address reprint requests to: Hideki Atsumi, MD, PhD, Department of Neurosurgery, Tokai University School of Medicine, 143 Shimokasuya, Isehara, Kanagawa 259-1193, Japan.

e-mail: h-atsumi@is.icc.u-tokai.ac.jp 